\title{
A game-theory modeling approach to utility and strength of interactions dynamics in biomedical research social networks
}

\author{
J. Mario Siqueiros-García ${ }^{1,2^{*}}$, Rodrigo García-Herrera ${ }^{2}$, Enrique Hernández-Lemus ${ }^{3}$ and Sergio Alcalá-Corona ${ }^{3}$
}

\begin{tabular}{l}
\hline *Correspondence: \\
jmario.siqueiros@iimas. \\
unam.mx \\
${ }^{1}$ IIMAS, Universidad Nacional \\
Autónoma de México, \\
Circuito Escolar 3000, Cd. \\
Universitaria, 04510 Mexico \\
City, Mexico \\
Full list of author information \\
is available at the end of the \\
article \\
J. Mario Siqueiros-García, \\
Rodrigo García-Herrera, \\
Enrique Hernández-Lemus, \\
Sergio Alcalá-Corona are \\
equally contributed at this \\
work.
\end{tabular}

*Correspondence: jmario.siqueiros@iimas. unam.mx

1 IIMAS, Universidad Naciona Autónoma de México, Circuito Escolar 3000, Cd Universitaria, 04510 Mexico

Full list of author information Sergio Alcalá-Corona are work.

\begin{abstract}
Purpose: Collaboration has become a cornerstone in biomedical research today. In contrast to physics which has a long history and experience in collaborative projects, biology is only recently becoming an evermore collaborative discipline. In this article we explore the effect of a collaboration network on the distribution of players having access to certain amount of resources from other players in the network and the distribution of the strength of interactions among them. We are interested in how they affect each other in the context of a network of scientific collaboration under the idea that while researchers are interested in maximizing their utilities, they also know that it is important to invest in building collaborative relationships.
\end{abstract}

Methods: We implemented two games played simultaneously: one for maximizing individual utility based on the iterated prisoner's dilemma; the other, a coordination game for maximizing the connection strength between players. We tested our simulation on a biomedical research community network in México and compared the results with Erdös-Renyí, a Watts-Strogatz small-world and Barabási-Albert topologies.

Results: Different topologies display different global utility and global strength of interaction distributions. Moreover, the distribution of utility and strength of interaction in the researchers network is similar to that of Barabási-Albert and Watts-Strogatz topologies, respectively.

Conclusions: Data related to Science, from co-authorships to Scientists' movility are increasingly becoming available. We think that the readiness of these sort of data is a great opportunity for scientists interested in the social dynamics of science, especially in the context of computational social science.

Keywords: Collaboration networks, Social networks analysis, Biomedicine, Game theory

\section{Background}

Collaboration has become a cornerstone in biomedical research today. In contrast to physics which has a long history and experience in collaborative projects, biology is only recently becoming an evermore collaborative discipline (Vermeulen et al. 2013). Biology has an interesting record in such matters because scientific collaboration means something different to different branches of biology: molecular biology has traditionally been a research activity of small laboratories (Cetina 1999; Strasser 2006), whereas in natural 
history there has been an exchange of data and samples since the seventeenth century (Wille 2012; Strasser 2012). Despite the differences in culture and practices, the Human Genome Project made collaboration a central feature of biology.

Nowadays it is widely acknowledged that collaboration takes many forms, from sharing of biological samples and biobanking to international groups in charge of helping research communities to harmonize and share their data (Vermeulen et al. 2013; Barrett et al. 2013; Federer et al. 2015; Karimi-Busheri and Rasouli-Nia 2015; Vallance et al. 2016; Warner et al. 2016). Sharing resources such as equipment, funds, and time is critical; building trust among scientists is fundamental (Bennett et al. 2010; Leite and Pinho 2017). Also, resources are mobilized in order to create strategic alliances.

The analysis of cooperation in scientific research has been the subject of a number of studies (Vermeulen et al. 2013; Newman 2001, 2004; Elango and Rajendran 2012; Hernández-Lemus and Siqueiros-García 2013; Strasser 2006, 2012). This is not surprising since cooperation and competition are quite important in today's academic success. Moreover, the quantitative analysis of cooperation in science also has a long history of more than 50 years (Franc 2012; Axelrod 2006). Particularly appealing to researchers studying this topic is the use of network analysis, especially in the area of bibliometrics and scientometrics such as in citation networks that date as far back as 1972 (Garfield 1972), co-authorship and collaboration networks also have a long tradition (Todorov and Winterhager 1991; Wagner et al. 2017).

The approach we follow here articulates game theory together with complex network analysis. Putting together game-theoretic modeling on complex networks is common in studies about the emergence of cooperation (Watts 1999; Szabó and Fáth 2007; Nowak and May 1992; Hisashi and Nowak 2006; Santos and Pacheco 2005; Santos et al. 2006; Suri and Watts 2011). However, in the context of science studies, it is far from being a unified, active field of research. There is no common set of questions and problems or a coherent body of models and theory aimed to understand the quirks and twists of cooperation in scientific communities. It is more earnest to say that work of this kind is rather scarce and focused around situations related to the social life of science, where sometimes models are run on collaboration networks because access to that kind of data is relatively easy.

Nevertheless, there is work done. For instance Wardil and Hauert have analyzed how collaboration happens within co-authorship networks (2015). Using the Physical Review journal historic database, these authors show the payoff differences between being the first author and the last author, where the first author is a cooperator that takes the highest load of work, while the last author is the laird that can benefit from the work of the former and get engaged in many other projects. They argue that cooperation in a multiauthored paper is a game more similar to "snow-drift" than to "prisoner's dilemma" since when co-authoring a paper everybody wins but each author pays a different price. Though this work takes into account the topology of the network as informative regarding cooperation patterns, the role of topology framing or fostering cooperation is not part of the study.

Game theory over complex scientific information and collaboration networks has also attracted attention. To have a better understanding of the market of scientific information Hanauske has applied Evolutionary Game Theory involving scientists as producers 
but also journals as disseminators. One of his models connects scientists' interest in maximazing their reputation and their decissions to publish in an Open Access Journal or in a journal that works under the traditional publication framework. He has also modeled game-theoretic interactions between scientists and journals concerning the decision of both players to go open access or not. Only this second model incorporates networks analysis (Hanauske 2012).

Another interesting behavior explored by game-theoretical modeling and complex networks has been done by Li and Cao (2003). These authors analyze the role of punishment in a science collaboration network. More precisely, they implemented an ultimatum bargaining model to understand the impact of the topology of a social network and the emergence of a population of punishers. In the ultimatum game, there are two players, one of them (the proponent) suggests to split a sum in two and its connected neighbor (the respondent) can decide to accept or reject the offer as it may be perceived more or less fair. If the respondent accepts, the sum is divided according to the offer. If the offer is rejected both players get nothing. According to the authors, the act of rejecting a selfish offer can be interpreted as a punishment since the player that rejects it is paying the cost of not receiving anything, but because of a selfish offer the proponent looses even more. Their results show that a phase transition will emerge in the population of punishers as the degree of selfishness increses among players. Moreover, they found a dependence relation between the tolerance to selfish behaviors and the topologies of networks.

In our model we explore the network effect on two issues: (a) the distribution of the amount of resources players can access from other players and, (b) the distribution of the strength of interactions among players as they interact. Also, in a similar vein to the work of $\mathrm{Li}$ and $\mathrm{Cao}$, we explore how resistant are the different topologies to defection as it becames harder for the population to cooperate. Particularly, we implemented two games played simultaneously: one for maximizing individual utility based on the iterated Prisoner's Dilemma (PD); the other, a coordination game for maximizing the connection strength between players. We are interested in how they affect each other in the context of a network of scientific collaboration under the idea that while researchers are interested in maximizing their utilities, they also know that it is important to invest in building collaborative relationships.

In the context of our model, utility represents access to resources shared by others. The value of the utility function for a player is the sum of the payoffs of playing prisoner's dilemma with its neighbors. In the other concurrent game players try to maximize the strength of their interactions by coordinating their strategy to that of their neighbors. Interaction strength is updated with no regard to cooperation or defection in the utility game. When both cooperate the interaction gets a positive payoff, when both defect, the interaction doesn't get affected; but if they do not coordinate, then the interaction loses. The payoffs resulting from the coordination games are not part of the utility function, however both are considered by the agent to decide whether to cooperate or defect.

From our anthropological fieldwork among biomedical research communities, their networks appear to be quite paradoxical: while they seem very hierarchical and only few researchers get the most of the profit of collective work, a great deal of collaboration is maintained. In other words, we are intrigued by the fact that for all the power and profit 
gain asymmetries, people are still willing to collaborate, build trust and remain loyal to their PI's and mentors. These two behaviors are explored in a biomedical research community in México. We believe that by implementing PD game and coordination game in a real world network is a way of capturing the essence of this problem. From our point of view this is an important contribution as we not only use data extracted from bibliometric source as most collaboration networks are studied, but that the founding question on which the model has been developed is empirically based in the sociology of the case.

The manuscript is structured in four sections. First we describe FOSISS, the main program for grants destined to applied biomedical research in México. This is the source of the database from which we created the researchers collaboration network. Next we describe our model and the different network topologies in which we explored it. We then present our results and discuss them. In the last section we draw some final remarks and conclusions.

\section{Biomedical research: CONACyT and FOSISS}

CONACyT or National Council of Science and Technology (Consejo Nacional de Ciencia y Tecnología) is the Mexican government entity in charge of promoting the development of science and technology. Among CONACyT's functions are to develop science and technology policies according to national needs and demands, to advise the different instances of government on scientific and technological topics, to promote the creation of research networks among the scientific community, to grant scholarships for masters and doctoral studies, and to manage different trusts intended to fund individuals and groups for scientific and technological research.

In the year $2002 \mathrm{CONACyT}$, along with other government agencies and entities, created sectoral funds to cover and equally promote research capacities of different areas such as energy, agriculture and health. Technological innovation is fostered by the generation of human resources and by helping research groups to consolidate. It is expected that the knowledge generated under the sponsorship of these funds will be the product of applied research that attends national public needs, and promotes economic growth. FOSISS or Sectoral Fund for Health and Social Security Research (Fondo Sectorial en Investigación en Salud y Seguridad Social) is one of such funds (González-Block et al. 2008).

FOSISS is constituted by CONACyT, SSA, IMSS and ISSSTE, ${ }^{1}$ all of them being the major public health providers and research institutions in the country. Every year CON$\mathrm{ACyT}$ opens a call for funds limited to a set of health research areas previously defined by a group of experts. Most applicants are public universities and research institutions, but eligibility is open to public and private health research sectors. From 2002 through 2013, there were 91 institutions funded that comprised 4988 researchers.

From these data some important considerations should be made clear. Scientists in the database take on the roles of principal investigators (PIs), associate researchers, postdoctoral associates, postgraduate and undergraduate students. Unfortunately, information on these roles is not specified in the database. We acknowledge the importance of this

\footnotetext{
${ }^{1}$ SSA is the acronym for Department of Health (Secretaria de Salud); IMSS is the acronym for Social Security Mexican Institute (Instituto Mexicano del Seguro Social); ISSSTE stands for Institute for Social Security and Services for State Workers (Instituto de Seguridad y Servicios Sociales de los Trabajadores del Estado).
} 
deficiency because researchers in our network act under different circumstances and we know that this diversity has a real impact on the structure and eventually on the dynamics of the network, as well as on the results of our model.

Our database includes the name of the project, the year it was approved for funding and the research area to which it was assigned. It specifies the names of PIs or the people responsible for the project and the names of collaborators. Researchers can be PIs in one project and collaborators on a different project. The institutional affiliation of all participants is included. Through this affiliation we determine the principal institution behind every project.

Even though curation and analysis work of this database is still going on, some relevant facts about the biomedical research can be said. Over the period of 12 years, 37 general research areas have been defined, the three most funded research areas are chronic and degenerative diseases, malignant neoplasms, and infectious and parasitic diseases. The least funded area is Ethics and medicine. The area with the most researchers is malignant neoplasms. Other areas of relevance for México are diseases related to poverty and Health and vulnerable groups (see Additional file 1).

From the institutions that have participated in a protocol funded by FOSISS, less than one fifth have been responsible for a project and more than $95 \%$ of them are Mexican, public institutions. There is also an important presence of foreign institutions as collaborators, most of them from the United States, though institutions from the UK, France, Spain, Netherlands, Colombia and Cuba are also in the database.

Besides the characteristics of the population there are some other boundary conditions that play an important role on the network topology and dynamics that motivated the development of our model. Biomedical research in México constitutes a vibrant community and collaboration is part of everyday work. However, México does not have public biobanks for research purposes (which are especially relevant for research in genomics, for example), and the current regulation on the access to biological samples such as tissue, cells, DNA, RNA, etc., is very general in scope (Murguia and SaruwatariZavala 2016). ${ }^{2}$ Something similar happens with data. There have been some attempts to create open data repositories for biomedical research, but they have not been established yet. Regulation on these subjects is still missing. Finally, technologically advanced equipment such as high throughput sequencers are kept by institutions with the highest research profiles and sometimes PIs manage them in a self serving way.

From our ethnographic work to date, we have been able to see that biological samples, data and technology can become instruments for negotiating collaboration. For example, among people involved in research projects, there are researchers that do not have direct access to samples, simply because their parent institution does not offer clinical services. Many of them are non medical doctors but chemists, biologists, physicists, and mathematicians. There is another group of researchers placed on hospitals that are able to do research and have access to biological samples from their own patients. It seems that this group is the most privileged one, and the one with the least pressure to establish collaboration at any cost. Finally, there is one more group formed by those who work

\footnotetext{
${ }^{2}$ Regulation exists regarding researcher-subject relations based on legal and ethical grounds. Also, all projects need to be approved by the Ethics Committee and IRB.
} 
as clinicians at small hospitals with no research infrastructure whatsoever. This group may have an interest in research and the way for them to become part of a project and be listed as authors in scientific papers is by giving researchers who do not have access to biological samples access to patients.

Due to these differences in the access to resources, researchers in general are compelled to build strategic alliances through which samples, data, technology and authorship, among other assets, become part of a constant flow through the network. Social and political capital, as well as concentrations of resources become fundamental tools for establishing fruitful collaborations.

\section{Methods}

Our model is based on the iterated version of the Prisoner's Dilemma (PD) and a coordination game instantiated on networks (Watts 1999). In our model, an agent's decision to cooperate or defect depends on a balance between utilities and the current strength of its collaboration relationships. Such balance reflects the overall success or failure of its strategies. We study the behavior of the system under different topologies, including a real-world network.

In our model, agents are embedded in a network with a varying number of neighbors. Following the traditional PD game, the strategy chosen by an agent and the strategy chosen by its neighbors will produce a payoff. Payoff follows the traditional PD rule: $T>R>P>S . T$ is for temptation to defect. It is the highest payoff and it takes place when the player defects and the other cooperates. $R$ is for reward for when both players cooperate. $P$ is for punishment for when both players defect. And $S$ is for suckers payoff, the worst outcome that takes place when the player cooperates but its neighbor defects. Utility is a property of agents in which payoff is accumulated.

\section{PD utility payoff matrix}

\begin{tabular}{lll}
\hline & Cooperate & Defect \\
\hline Cooperate & $R, R$ & $S, T$ \\
Defect & $T, S$ & $P, P$ \\
\hline
\end{tabular}

The strength of the interaction, represented by $w$, is a property of the link between two agents and gets updated according to the payoff matrix of a coordination game. In the $w$ matrix, the highest value goes to an edge when both agents cooperate, getting an $R$ for reward; if one of them defects, the connection gets weaker getting $P$ for the collaborative connection being punished. If both agents defect, the value $w$ doesn't change, which means that agents didn't interact or that the interaction gets nullified $N$. In this game, the best action for any agent is to coordinate with its neighbor, either because it wins or because it doesn't lose.

w payoff matrix

\begin{tabular}{lll}
\hline & Cooperate & Defect \\
\hline Cooperate & $R$ & $P$ \\
Defect & $P$ & $N$ \\
\hline
\end{tabular}


After each game, the agent adds up utility $(u)$, which is the sum of the payoffs following the PD matrix. A pair of neighbors will add or substract from the strength of their interaction $\left(w_{j i}\right)$ as they coordinate or not, being $w$ also cumulative. We measure global utility and strength of interaction for the whole network. Global utility $U$ is the sum of all individual utilities and global strength of interaction or $W$ is the sum of every pair of agents' links $w$. Strength of interaction may have different meanings. From an empirical point of view, it simply means the number of times two researchers have collaborated together in a common research project. From a sociological and qualitative point of view, if these two scientists have been willing to collaborate more than once, it may signify some form of "loyalty" among them. But this may not be the only possible interpreation since it can be different for each case. Since the strength of interactions can be sociologically interpreted in so many ways, we decided to just quantify the times two researchers collaborate, and by doing this avoiding any generalized interpretation of the quality of the interactions among researchers.

It should be noted that the same actions or behaviors work for both $u$ and $w$. There are two reasons for this decision in the design of the model. The most general one is that we believe that in the real world, actions such as cooperating and defecting affect the strength of the interaction among people. The second one is that we think that selfishly maximizing access to resources and strengthening relationships are opposing forces acting on the same set of behaviors. The actions of an agent imply a trade-off in which defecting may increase its utility at the expense of its collaborative relationships. If collaborators have nourished their relationships, they might be strong enough to endure occasional defection. Cooperating may build up relationships but it can be expensive for the player.

\section{Model update}

All networks are initialized equally. The number of nodes for every network is 4122 , the same as in the FOSISS network. The same utility is given to every agent and all edges are assigned the same weight. In the case of the FOSISS network, edge weight is given by the number of collaborations among researchers, utility remains the same for all nodes as in the other networks.

The probability for an agent to cooperate or defect depends on a number $(\eta)$ that refers to a historical balance between average utility $\left\langle u_{i}\right\rangle$ and the average strength of the connections with its neighbors $\left\langle w_{i j}\right\rangle_{j}$. It is modeled so because we assume that whatever the result in utility or strength of interaction, as long as one of them increases, the player will be confident in the strategy followed so far. In every simulation step, each agent will independently play both games with every neighbor. Nonetheless, the agent sets its decision to cooperate or defect beforehand by assessing the overall situation of its relationships. This may sound odd, since one may think that a person decides for each relationship separately. However in this way players are optimizing access to resources and the strength of interactions to their neighbors at the same time, since their state is set by considering both together. The strength of interaction is updated independently for each pair of agents. In a single play some interactions might be weakened, some may get strengthened and others may remain unaffected. Moreover, in this model, history is kept in the network as strength of interactions and it conditions the agents disposition 
to cooperate or to defect. As the sayings go: Burnt children dread fire; A bad dog is not born but made.

$\eta$ is calculated as:

$$
\eta_{i}=\frac{\left\langle u_{i}\right\rangle+\left\langle w_{i j}\right\rangle_{j}}{2}
$$

For the agent to decide whether to cooperate or not, $\eta$ is compared to a global parameter $v$. This is a parameter that represents the minimum value of $\eta$ that a player must achieve in order to cooperate. If the agent's $\eta>v$, then he will cooperate, otherwise he will be suspicious and will defect. In this way, as we arbitrarily control the value of $v$ we can identify the values of $\eta$ where the population stops cooperating and begins to defect; also it makes possible to see how this transition takes place for the different network topologies.

Our simulation was tested on an Erdös-Renyí, a Watts-Strogatz small-world and Barabási-Albert topologies, as well as on the real biomedical research collaboration network. The simulation was run in a synchronous manner, in which all agents update their behavior simultaneously.

We ran two different experiments. In the First Experiment we simulated different values of the parameter of confidence $v$. With this experiment we were able to see how the number of cooperators, utility, strength of interactions among agents and the ratio of shifting state population would change in the range of the parameter of confidence. The states of the agents were the same at initialization, for all values of $v$. Since the model is deterministic, it will return the same result if run under the same conditions.

The Second Experiment consisted in running the simulation under the same value of the parameter of confidence $v$ but randomizing the initial states of the agents. This would show that the system converges to a global state. For every network, the simulation was run 100 times and results were averaged.

\section{Implementation of the model in different topologies}

We built three classical topologies for networks besides the FOSISS network, their parameters are shown in the following table.

\begin{tabular}{lllll}
\hline Topology & $\boldsymbol{m}$ & $\langle\boldsymbol{k}\rangle$ & $\langle\boldsymbol{C}\rangle$ & $\langle\boldsymbol{I}\rangle$ \\
\hline Erdös-Rényi & 25,591 & 12.4 & 0.003 & 3.6 \\
Watts-Strogatz & 206,100 & 100 & 0.7 & 3.4 \\
Barbási-Albert & 183,465 & 89 & 0.06 & 2.13 \\
FOSISS & 23,391 & 11.39 & 0.87 & 5.49 \\
\hline
\end{tabular}

\section{Erdös-Renyí}

Erdös-Renyí networks (1959) (random networks) are constructed by randomly selecting a pair of $N$ possible nodes and attaching them with an edge, given a probability $p$, as long as there is no edge between them. The result is a Poisson distribution for connectivity of nodes $P(k)$, where each node has a degree quite close to the average $\langle k\rangle$. Also for this type of network, average clustering coefficient $\langle C\rangle$ is small, actually it is equal to $p$ (the probability of connecting two nodes) and the average shortest path length $\langle l\rangle=\frac{\ln N}{\ln \langle k\rangle}$. 


\section{Small-world}

Watts-Strogatz networks (1998) (small-world networks) are in a regime between a fully regular grid (lattice) and a random network (Erdös-Rényi). In order to build them, a node is chosen from a lattice (a ring) and the edge that connects it to its nearest neighbor in a clockwise sense. With probability $p$, this edge is reconnected to a node chosen uniformly at random over the entire ring, with duplicate edges forbidden; otherwise the edge is left in place. This process is repeated by moving clockwise around the ring, considering each node in turn until one lap is completed. Next, the edges connect nodes to their second nearest neighbors clockwise. As before, each of these edges is randomly rewired with probability $p$. This process continues, circulating around the ring and proceeding outward to more distant neighbors after each lap, until each edge in the original lattice has been considered once. The main characteristic of these networks is that the average shortest path length is small and grows as $\log (N)(\langle l\rangle \sim \log (N))$. Also, the average clustering coefficient $\langle C\rangle$ remains large in terms of $p$. For $p<0.1,\langle C\rangle \sim 1$.

\section{Barbási-Albert}

Barbási-Albert networks (1999) (scale-free networks) are generated by adding new nodes to a network. Each new node is added connecting it to an existing node with a probability proportional to the degree $k$ (connectivity) of each node (preferential attachment). The result is a power law distribution for connectivity of nodes $P(k)$ where few nodes have many connections and the most have very few connections. Furthermore these networks are also small world networks, showing a quite small $\langle l\rangle$.

\section{FOSISS: biomedical research community network}

The biomedical research network on which we are running our model was generated with data from collaborative projects. Our data was obtained from CONACyT and includes twelve years of information of FOSISS grants. Data included names of principal Investigators, collaborators, research topics, etc. The network we are using here has researchers as nodes and edges represent the connection of two scientists when they collaborate on the same project. Edges are also weighted according to the number of projects shared by any pair of scientists.

\section{Results}

In this section we present the main results of the study, namely the topological structure of the underlying network models, the dynamics of the games under different parameters and network topologies and the distribution of utility and of the strength of interaction resulting from playing the games in all the different scenarios considered, including the real FOSISS network.

FOSISS network summed up a total of 145 components or subnetworks, but we ran the model on the giant component made up of 4122 researchers, and 23,391 edges. The giant component was analyzed using Cytoscape and is shown in Fig. 1. Results show that it is a well integrated network, with a clustering coefficient $\langle C\rangle=0.870$, an average shortest path length of $\langle l\rangle=5.493$ and a density of $p=0.003$. Such properties recall a small-world topology (Watts and Strogatz 1998), and a great deal of self-organization when compared to a random network with the same density and number of nodes. 


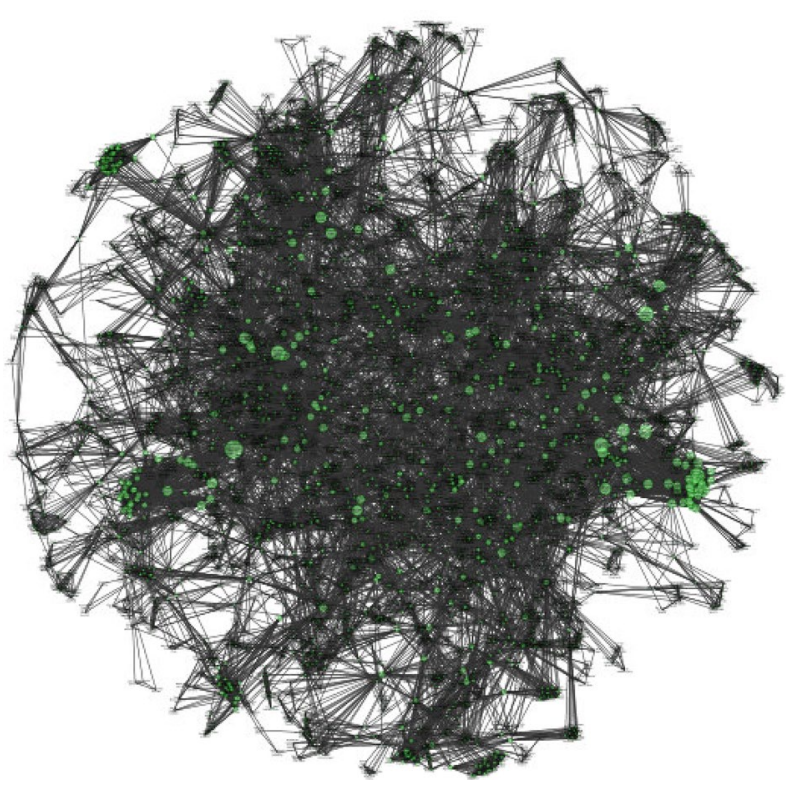

Fig. 1 Biomedical research collaboration network (FOSISS) giant component

Network centralization is 0.023 , since there are no visible researchers that play as hubs in the network. Nevertheless the network heterogeneity is 0.873 , which means that the network is highly hierarchical. When the degree distribution is analyzed, degree decreases as a power-law with an exponent of 1.7, similar to other social networks described as scale-free topology networks (Barabási and Albert 1999). Finally, the average number of neighbors of each node is 11.39 (Shannon et al. 2003).

Other results to report are those of the dynamics of different variables as the parameter of confidence $v$ changes. The most salient result is that for $v$ between 0.19 and 0.24 , there is an apparent phase transition in all different topologies and for all the different variables. It is worth noting that the shape of the phase transition-like behavior is different according to the topology of the network under study. When $v$ is between 0.0 and 0.2 , that is, when there is no space or a very short one for suspiciousness, all agents cooperate, when $v$ is above 0.25 , all agents defect. Utility, strength of interactions, and changing state population replicate that same behavior for the same limits.

In Fig. 2, we present how the number of cooperators in the population change as $v$ changes. In the Erdös-Renyí, network, between 0 and 0.18 approximately, all agents converge to a cooperative behavior, from 18 to 20, convergence to cooperative state takes longer but eventually all agents are cooperating. Close to $v \approx 0.21$ there is a sharp fall to a point in which around half the population is cooperating and the rest is defecting. Reaching $v \approx 0.25$ there is another sharp fall of cooperators and all agents turn into a defecting state.

In the case of the Watts-Strogatz small-world network, the whole population remains cooperating for ranges between 0 and 0.2 but as it gets closer to 0.2 , more time is needed for the population to become full of cooperators. In $v \approx 0.2$ the cooperators will represent only half of the population and such number of them will be constant up to $v \approx 0.25$, forming a short plateau. From $v \approx 0.25$ to $v \approx 0.6$ cooperators will be present 


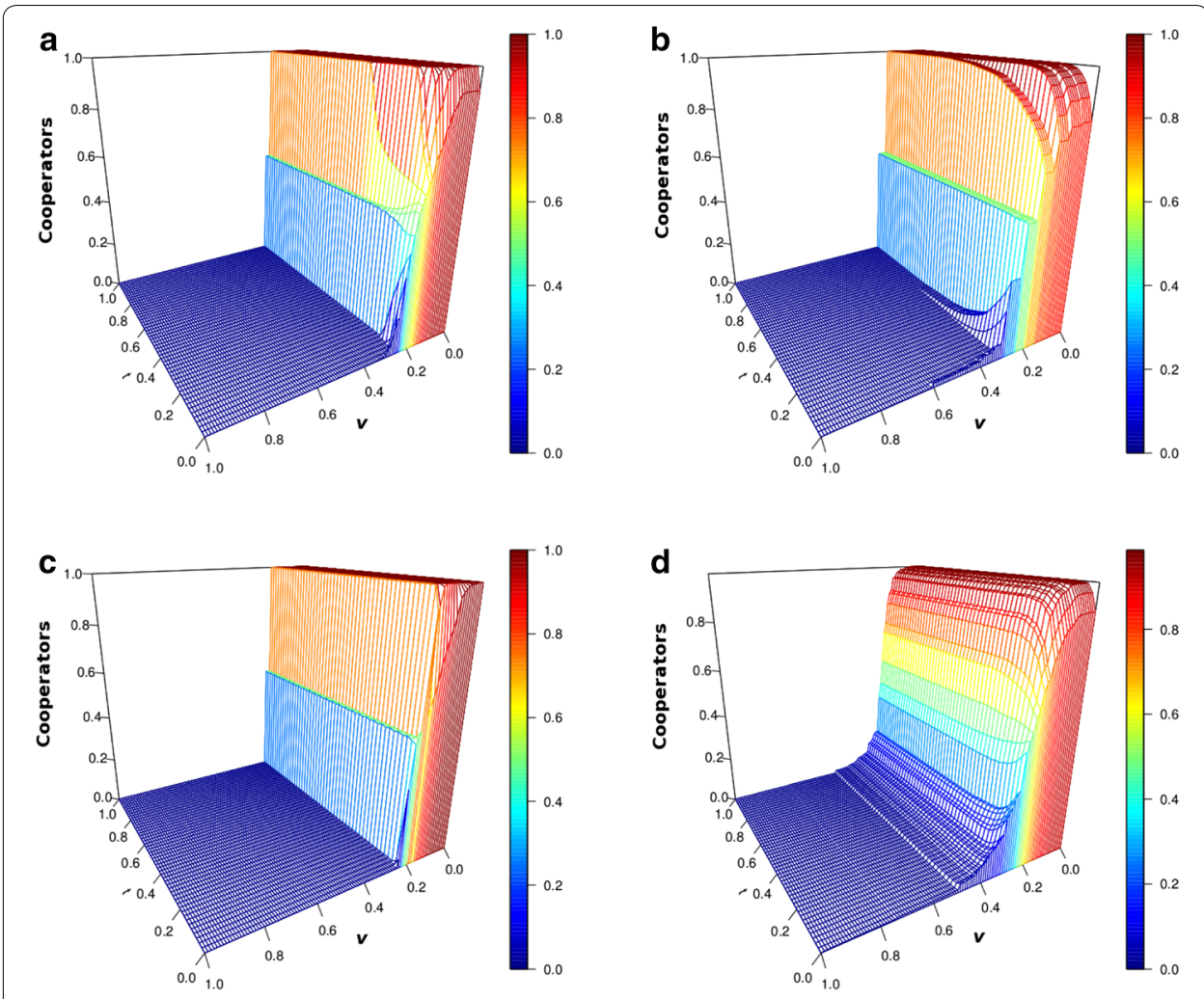

Fig. 2 Ratio of cooperators as a function of the parameter of confidence and time during First Experiment. Topologies are: a Random, Erdös-Rényi. b Watts-Strogatz. c Barabási-Albert. d FOSISS

at the beginning of the simulation but will diminish as time goes on. In the case of the Barabási-Albert network, there is a sharp decrease in the number of cooperators when the threshold of $v \approx 0.2$ is crossed, but stays constant over time. Such behavior is present for a very short range of $v$, and before $v \approx 0.24$, cooperators disappear for the rest of the values of $v$. Finally, FOSISS network behaves similarly to the other networks in that there is a fall in the number of cooperators close to $v \approx 0.2$. In contrast with the other networks, the FOSISS network lacks the sharp reduction of cooperators, instead this population declines smoothly and progressively; especially, when it reaches a $v \approx 0.25$, cooperators decrease in a less dramatic manner all the way to $v \approx 0.5$. It is also noteworthy that from $v=0$ to $v \approx 0.5$ the number of cooperators converge to a certain degree and stays constant for the rest of the simulation.

Utility and strength of interactions dynamics under different $v$ are similar to the phase transition found before. Figures 3 and 4 show that there is a drop in utility and strength of interactions according to the drop in the number of cooperators. Erdös-Renyí and Barabási-Albert networks are quite similar in the way these variables fall in two steps, the first one at $v \approx 0.2$ and the next one at $v \approx 0.23$. The fall is even sharper in the BarabásiAlbert topology. Utility and strength of interactions phase transition in Watts-Strogatz network is significantly more staggered compared to the former networks. In the case of utility, there is a region in the limits of $v \approx 0.25$ and $v \approx 0.3$, before utility goes to 0 , in which it remains low but stable over time. In general, strength of interactions follows 

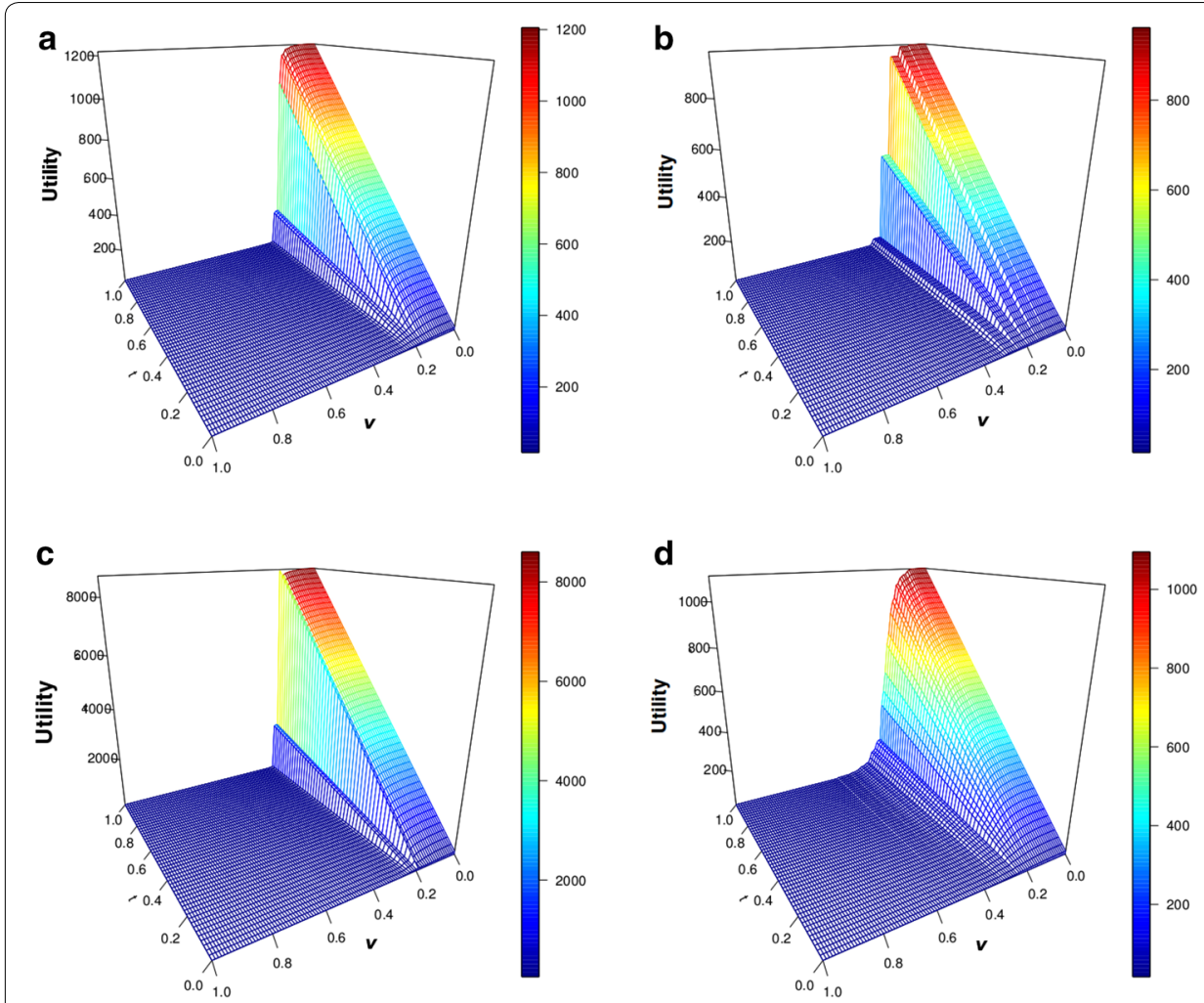

Fig. 3 First Experiment's utility $U$ dynamics as a function of the parameter of confidence $v$ and time. Topologies are: a Random, Erdös-Rényi. b Watts-Strogatz. c Barabási-Albert. d FOSISS

the same pattern as utility but in the same $v \approx 0.25$ and $v \approx 0.3$ strength of interactions grows to a value that is higher than the one given by default but soon starts to decrease as the simulation runs. For the FOSISS network, utility and strength of interactions fall quite steeply but smoothly, without sharp cuts. Between $v \approx 0.23$ and $v \approx 0.28$, utility and strength of interactions start at their lowest, but there is a slight increase in both of them.

We also measured the number of agents shifting states-between cooperating and defecting-under different $v$ values. We found that for all networks there is a critical point around $v \approx 0.2$ in which all agents are shifting states. For Erdös-Renyí and Barabási-Albert networks, for this region, agents never settle to a single state. Contrary to the former cases, the number of shifting agents decreases considerably for the WattsStrogatz and FOSISS networks, and find an equilibrium state. Once the the limits of this region are crossed as $v$ increases, the number of agents shifting states falls to 0 and all nodes become defectors (Fig. 5).

Central to our argument are the differences in utility and strength of interactions distribution at the end of the simulation, for every topology. We found that utility distribution for the FOSISS network resembles quite accurately the distribution of utility in the Barabási-Albert network.

The distribution of utility on each topology is induced by the degree distribution. This is so since a given agent (node) will interact with its neighbors to either cooperate or 

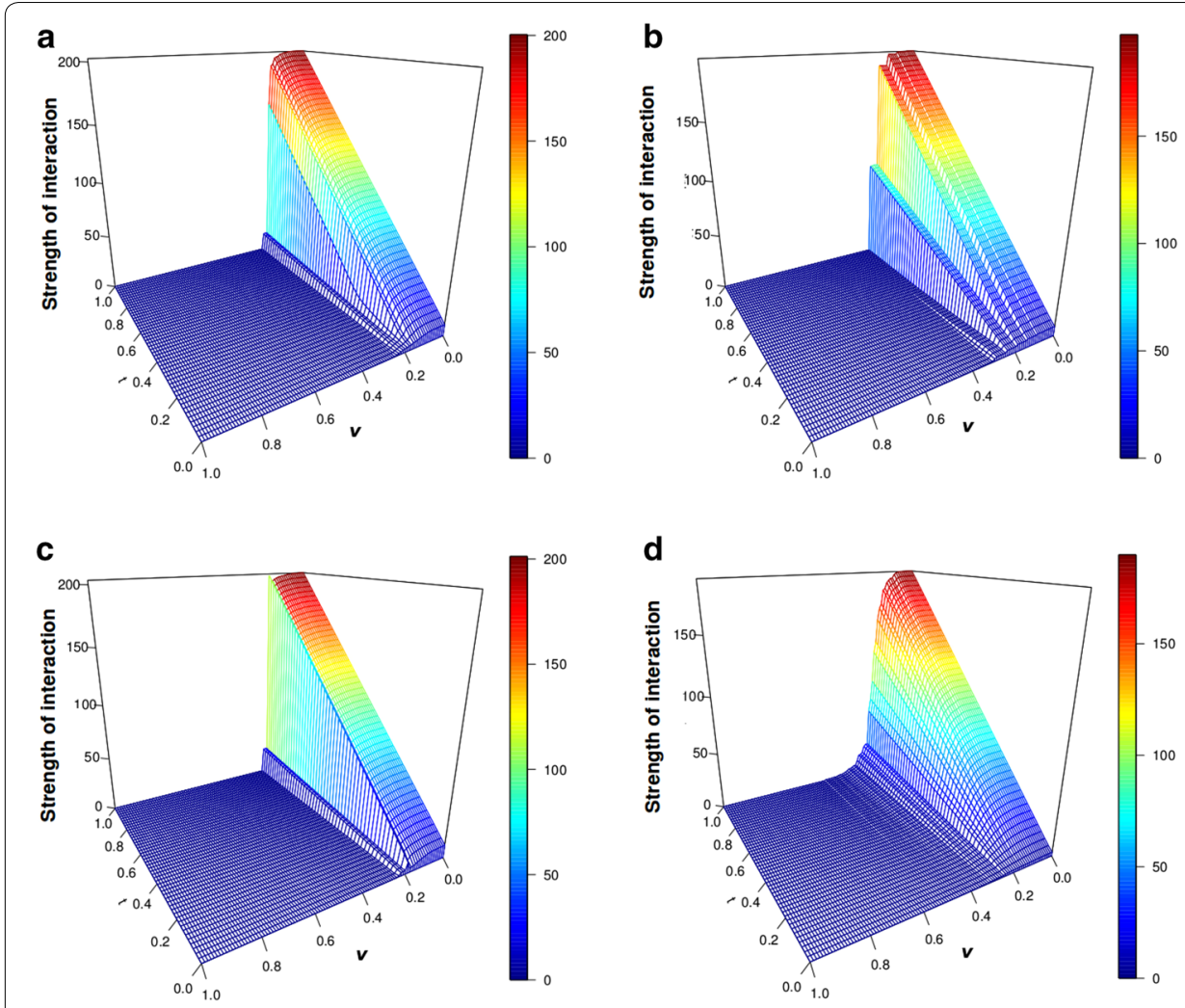

Fig. 4 Strength of interactions $w$ dynamics as a function of the parameter of confidence $v$ and time during First Experiment. Topologies are: a Random, Erdös-Rényi. b Watts-Strogatz. c Barabási-Albert. d FOSISS

defect in such a way that connectivity influences the number of events played and thus the likelihood of increasing its corresponding utility. For instance, utility distribution in the random Erdös-Rényi network displays a normal-like curve. The algorithm that generates this kind of topology, assigns to every node the same probability of connecting with any other node, which produces a poissonian degree distribution (Erdös and Renyí 1959). Since the Watts-Strogatz degree distribution is described by a function that is midway between a random distribution and a scale-free network (Barrat 2000) one may expect also an intermediate behavior of the utility distribution. This assumption seems to be fulfilled by the distribution in Fig. $6 \mathrm{~b}$.

The resemblance of the degree distribution and utility distribution also holds for the Barábasi-Albert network. As mentioned in the methods section, the degree distribution of a Barábasi-Albert topology follows a power-law that describes the fact that there is a small number of nodes with large $k$ and most nodes have a small $k$ (Barabási and Albert 1999). As it is shown in the following figure, most utility is concentrated in a few number of agents, while most agents have a small amount of it. This is consistent with other research in which concentration of resources, fame or citations in science decreases as a power-law (Simon 1955; Price 1965; Merton 1968). FOSISS network utility distribution is also skewed to the left, similar to that of the Barabási-Albert network.

Regarding strength of interactions distribution, the Erdös-Rényi random network displays a normal distribution of strength of interactions, as expected. Again strength 

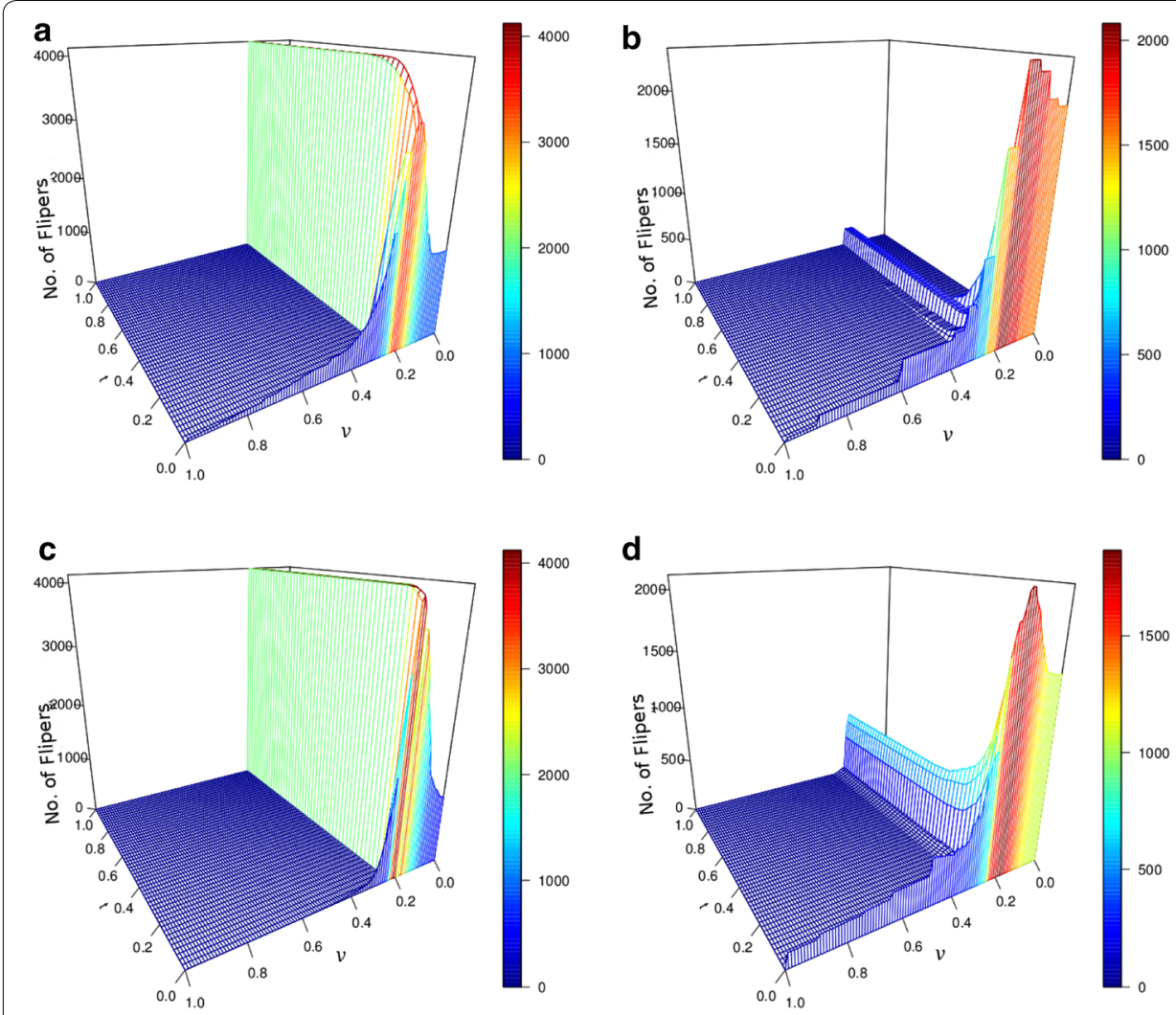

Fig. 5 Shifting population between cooperators and defectors as a function of the parameter of confidence $v$ and time during First Experiment. Topologies are: a Random, Erdös-Rényi. b Watts-Strogatz. c BarabásiAlbert. d FOSISS

of interactions values are highly influenced by the corresponding degree distribution (Fig. 7a). However, in the Watts-Strogatz network topology (Fig. 7b), the strength of interactions distribution is a highly asymmetric bimodal, with a really low frequency mode of low strength of interactions and a high probability mode for high strength of interactions. A possible explanation for this phenomenon is that under network topologies maximizing inter-node communication (by minimizing the average distance between nodes) such as the Watts-Strogatz, strength of interactions is favored both among the cooperators (constituting the majority of players) and the defectors.

The Barabási-Albert network (Fig. 7c) also presents a symmetric unimodal distribution with values higher (on average) than those of the Erdös-Rényi random network, this may be the effect of increased communication due to more efficient network navigability. Interestingly, the network corresponding to the real FOSISS collaborations (Fig. 7d) is an asymmetric unimodal distribution in which moderate to high values of strength of interactions are more likely. We hypothesize that this effect is also due to the communication properties of the network.

An interesting feature of highly communicated networks (characterized by high values of clustering coefficient) is the fact that certainty among players seems to be enhanced, that is, in such networks the rate of strategy change is significantly lower (and smoother) than in poorly connected networks. This is another instance in which easier 


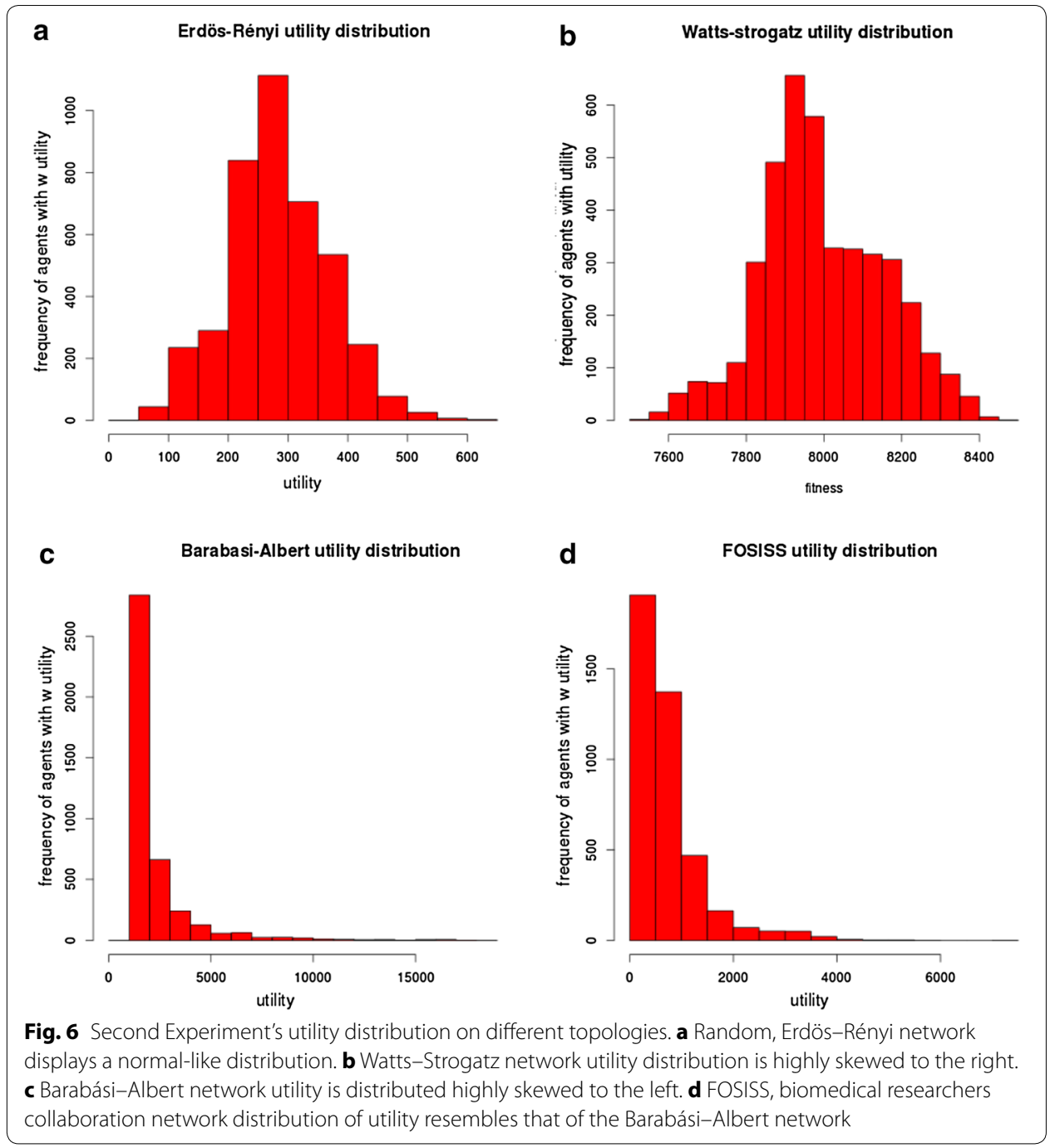

communication (i.e. lower average minimum path lengths) leads to better performance of the whole collaborative research system.

\section{Discussion}

In this work we have analyzed the influence of parameters given by the underlying social structure of a science collaborative network on collective strength of interactions and utility dynamical behavior, based on a class of iterated PD and coordination games. Such parameters include mainly local and global connectivity like the degree centrality and average clustering coefficient, as well as communication patterns.

Under the assumptions given by the model, we were able to notice that, in general, communication within the social collaborative networks has a positive correlation with average strength of interactions between the individuals partaking in the games and also with the global collective utility (given by the sum of the individual payoffs). The better the communication among players, the higher the strength of interactions and the utility leading to an optimized functioning of the whole scientific collaboration system. 


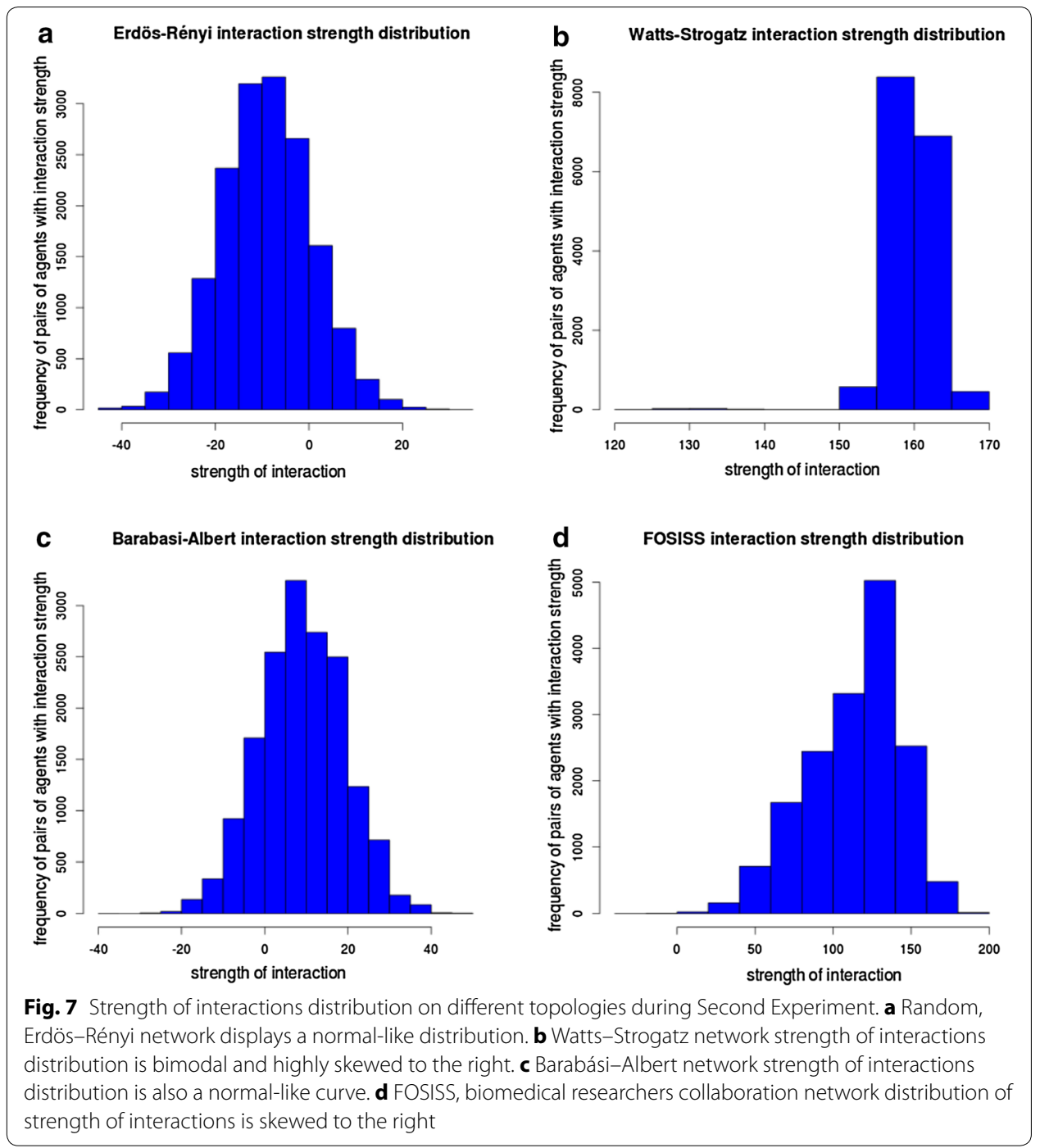

This is an important result that may be useful for scientific policy planning and may set a foundation for the optimal use of social networks in scientific collaboration as a means to improve the relationships among collaborating peers and ultimately the performance of research systems. We obviously need more qualitative work in order to validate these results from a sociological and anthropological perspective.

In the area of complex networks and game theory, it has being affirmed for a long time that scale-free networks promote the evolution of cooperation. Part of this property has been attributed to the presence of hubs (Santos and Pacheco 2005; Szolnoki et al. 2008; Du et al. 2008; Li and Cao 2003; Yang et al. 2009; Chuang et al. 2009; Liu et al. 2010; Hong-Yan and Jun-Zhong 2011; Li and Duan 2014; Li and Yong 2015; Ichinose and Sayama 2017). Perhaps less attention has been place on the persistence of cooperation and the the role played by the clustering coefficiente in scale-free and small-world networks (Kim et al. 2002; Masuda and Aihara 2003; Wu et al. 2005, 2006; Jian-Yue et al. 2006; Fu et al. 2007; Zhang et al. 2007; Thibert-Plante and Parrott 2007; Chen et al. 2008). This is where our result comes in. 
The two main outcomes of our model are the smoothness in the phase transition-like behavior for different parameters and the distribution of utility and strength of interactions in the FOSISS network compared to other networks and their topologies. We are certain that the particular structure of FOSISS network is playing a central role in the results and because of that we would like to discuss it a little bit further. FOSISS network is highly hierarchical according to its heterogeneity of 0.873 . Because of it, one would expect to find the presence of important hubs (Jun et al. 2008), that is, a few researchers control the whole network, as has been reported in some elsewhere (Yousefi-Nooraie and Akbari-Kamrani 2008). Surprisingly, FOSISS network centralization is very low 0.023 , i.e. there are no researchers that centralize the majority of connections. Our guess is that the network is composed of many small communities or groups with a central researcher or Principal Investigator (PI). If this is the case, it means that those groups have a very hierarchical structure as well. This is similar to what has been reported by others about the heterogeneity in small-world networks, in which the combination of certain amount of hierarchy and small-worldness makes cooperation to peak over compared to the evolution of cooperation in homogeneous and the most heterogeneous networks (Fu et al. 2007). For the FOSISS netwok, this property would enhanced cooperation among players, eventhough the distribution of utilities are skewed towards a handful of researchers.

Under such structure, when the clustering coefficient is considered, it can be said that groups are also well connected but that inter-group connections are sparse. In other words, individual groups are strongly connected within but the network as a whole is supported by a small number of links. This is not an uncommon pattern in social networks. It has been claimed that this form of structure has been evolutionarily advantageous (Turchin 2015). Moreover, research groups internal cohesion may be related to Wardil and Hauert idea that collaboration among scientists-at least in the case of multi-authored articles, is more similar to a "snow-drift game" than to a PD game, since in the former everybody wins or everybody pays a cost but the cost are not evenly distributed among the players.

We came by the idea that intra-networks are stronger than inter-networks, from another study about scientific collaborations based on co-authorships (HernándezLemus and Siqueiros-García 2013). In the cited reference, an apparently well integrated community was found (high clustering coefficient and a very short characteristic path length). Such integration was mostly superficial, since it depended on the presence of external collaborators from other research institutions (most of them from overseas). Removing these external collaborators brakes down the network into small subgraphs that worked independently. Remarkably, those subgraphs corresponded to the real groups of that research center. What is more, several groups had a hierarchical structure as the one we suspect is common in FOSISS subgraphs. The results showed that collaboration was poor between groups but strong among the members of each group, and that collaboration among groups doesn't emerge bottom-up, instead it seems to be promoted from the top, from the administrative authorities.

We believe that the situation just referred is also true for the whole biomedical research community in México. The amount of PIs who have also been collaborators in other projects is about one fifth of the total number of participants. This is a number big 
enough to connect the whole network in one giant component. Yet, due to the topological characteristics of the FOSISS network, it appears to be the case that researchers can be the leaders in one project and collaborators in a different project of its own research group. CONACyT's funding policies makes it impossible for a researcher to get a grant from a fund if that researcher has an ongoing project with a grant from that same fund. That is, a researcher can ask for a grant from FOSISS if and only if at the moment he doesn't already have one. This policy has lead researchers from the same group to ask for grants from the same fund in order to rise their budgets. A consequence of this behavior is that group interactions get reinforced, but inter-group connections not necessarily so.

As is common among scientific communities in biomedical research, PIs play a central role in the network. Strong PIs and well connected groups seem to be somehow responsible for the high levels of strength of interactions and the centralization of utility in our simulation. As for what seems to be phase transitions, in the case of FOSISS networks, these are smoother than those in the other networks with different topologies, even for those with a small-world topology. We think that this behavior is also the result of the hierarchical structure already mentioned. If this is true, strength of interactions is first lost in the edges that link different groups and then in the edges that connect members of the groups.

Connections between groups would not be as dense as those inside the groups, which means that there would not be enough information on the behavior of one group regarding its neighbors to constrain them as it seems to happen with individual researchers inside their communities. Nevertheless, if values of the parameter of cooperation $v$ keep increasing and it becomes more difficult to strengthen interactions, then strength of interactions begins to diminish inside groups. And yet, the smoothness observed in the small-world network and FOSISS network may be attributed to the power of the clustering coefficient. As has been referred by Li and Cao $(2003,2011)$, and Thibert-Plante and Parrott (2007) for scientific collaboration networks, high clustering coefficient allows the system to resist the invasion of non-cooperators.

Another issue that we would like to mention about FOSISS network topology is that it is not a robust collaboration network. At the level of groups, nodes might be well connected and consolidated but at the level of the network, they could be no more than an aggregate of individual groups. A robust network would be resistant to changes in the connections between groups but in the case of FOSISS, it seems that the network would brake down into small research groups by cutting some edges, as it happened in our coauthorship collaboration network (Hernández-Lemus and Siqueiros-García 2013). The lack of robustness might be indicative of the fact that resources stay inside the groups, that is, they do not circulate through or articulate different communities. For example, one may think of certain expensive technologies for genomic research that could be bought once and shared among research groups, however, this doesn't seem to happen very often. There are some other consequences, such as low communication among groups, atomization of practices and know-hows, redundancy in equipment tenancy, difficulties in implementing community-wide infrastructures such as biobanks, etc. 


\section{Conclusions}

Our future work is based on the results presented here. We would like to identify researchers in our simulations and corroborate their situation in the model and in the real world. We are also interested in going back to the field and interviewing those groups with an interesting behavior found in our simulations, we would probably follow a similar strategy as the one developed in (Hara et al. 2003). Another idea we would like to follow is related to the parameter $v$. As it is now implemented in the model, $v$ is an external parameter to the system but in future work we would like to explore the possibility of $v$ being controlled by the dynamics of the system. The assumption behind this idea is that society self-imposes norms and it dynamically regulates how difficult is for its members to comply. In our model we used this parameter to control how hard or easy is for a player to cooperate. Finding communities beyond the level of the groups is an important task. We think that there are many possibilities that emerge from the integration of different methodologies. Moreover, studying social processes in science is particularly attractive due to the amounts of data already available that can be easily collected. This is a privilege because simulations can be designed on real world data, something that only very recently has become possible (Barabási 2012).

No doubt social sciences are becoming more "computational" (Conte 2012). This can be seen everywhere, but curiously enough, disciplines like physics and computer science are moving towards the social sciences and not so much the other way around. It might be the case that the pioneering disciplines in the computational social sciences will set the agenda, an agenda that will apparently be mostly based on taking advantage of big data and on hypothesis-free approaches. We believe that the social sciences have important questions that should be added to that agenda and those questions may not be answered only by big data techniques but they may require creating models and simulations in the style of the best hypothesis-driven research.

\section{Additional file}

Additional file 1. FOSISS research areas.

Authors' contributions

JMSG jointly conceived the study with RGH and EHL, designed and implemented the simulation model with SAC. All authors read and approved the final manuscript.

Author details

1 IIMAS, Universidad Nacional Autónoma de México, Circuito Escolar 3000, Cd. Universitaria, 04510 Mexico City, Mexico. ${ }^{2}$ LANCIS-IE, Universidad Nacional Autónoma de México, Ciudad Universitaria, 04510 Mexico City, Mexico. ${ }^{3}$ Instituto Nacional de Medicina Genómica, Periferico Sur 4809, 14610 Mexico City, Mexico.

\section{Acknowledgements}

We acknowledge the help of Ma. del Pilar Galarza and Francisco Allende for their work curating databases of researchers. We are grateful to Be Luecke for copy editing the manuscript. We would also like to thank to the reviewers for their comments and suggestions

\section{Competing interests}

The authors declare that they have no competing interests.

\section{Funding}

The authors gratefully acknowledge support by grants: CB-222220-R/2013 and CB-179431/2012 (Consejo Nacional de Ciencia y Tecnología), PAPIIT A301016 (UNAM). We would also like to acknowledge CONACyT for letting us access their database. 


\section{Publisher's Note}

Springer Nature remains neutral with regard to jurisdictional claims in published maps and institutional affiliations.

Received: 13 January 2017 Accepted: 25 April 2017

Published online: 16 May 2017

\section{References}

Axelrod R (2006) Evolution of cooperation, Revised edn. Basic Books, Cambridge

Barabási AL (2012) The network takeover. Nature 8:14-16

Barabási AL, Albert R (1999) Emergence of scaling in random networks. Science 286:509-512

Barrat M, Weigt M (2000) A; Weigt. On the properties of small-world network models. Eur Phys J B 13(3):547-560

Barrett T, Wilhite SE, Ledoux P, Evangelista C, Kim IF, Tomashevsky M et al (2013) NCBI GEO: archive for functional genomics data sets-update. Nucleic Acids Res 41(D1):D991

Bennett LM, Gadlin H, Levine-Finley S. Collaboration \& team science: a field guide. 2010

Chen Y, Qin SM, Yu L, Zhang S (2008) Emergence of synchronization induced by the interplay between two prisoner's dilemma games with volunteering in small-world networks. Phys Rev E 77(3, 1):032103

Chuang L, Jian-Yuan J, Xiao-Jie C, Rui C, Long W (2009) Prisoner's dilemma game on clustered scale-free networks under different initial distributions. Chin Phys Lett 26(8):080202

Conte R, Gilbert N (2014) Manifesto of computational social science. Eur Phys J Special Top 214(1):325-346

Du WB, Zheng HR, Hu MB (2008) Evolutionary prisoner's dilemma game on weighted scale-free networks. Phys Stat Mech Appl 387(14):3796-3800

Elango B, Rajendran J (2012) Authorship trends and collaboration pattern in the marine sciences literature: a scientometric study. Int J Inf Dissem Technol 2(3):166-169

Erdös P, Renyí A (1959) On random graphs. Publ Math 6:290-297

Federer LM, Lu YL, Joubert DJ, Welsh J, Brandys B (2015) Biomedical data sharing and reuse: attitudes and practices of clinical and scientific research staff. PLOS ONE 06:10

Fu F, Liu LH, Wang L (2007) Evolutionary prisoner's dilemma on heterogeneous Newman-Watts small-world network. Eur Phys J B 56(4):367-372

Garfield E (1972) Citation analysis as a tool in journal evaluation. Science 178:471-479

González-Block MA, Mercado FJ, Ochoa H, Rivera H (2008) Utilización de la investigación por gestores de salud en México: diagnóstico de la capacidad y propuestas de fortalecimiento. Salud Pública de México, Mexico

Hanauske M (2012) Evolutionary game theory and complex networks of scientific information. In: Scharnhorst A et al (eds) Models of science dynamics, understanding complex systems series. Springer, Berlin, pp 159-191

Hara N, Solomon P, Kim S, Sonnenwald D (2003) An emerging view of scientific collaboration: scientists' perspectives on collaboration and factors that impact collaboration. J Am Soc Inf Sci Technol 54(10):952-965

Hernández-Lemus E, Siqueiros-García JM (2013) Information theoretical methods for complex network structure reconstruction. Complex Adapt Syst Model 1:8. doi:10.1186/2194-3206-1-8

Hong-Yan C, Jun-Zhong Y (2011) Organization of the strategy pattern in evolutionary prisoner's dilemma game on scalefree networks. Chin Phys Lett 28(6):060201

Ichinose G, Sayama H (2017) Invasion of cooperation in scale-free networks: accumulated versus average payoffs. Artif Life 23(1):25-33

Jian-Yue G, Zhi-Xi W, Zi-Gang H, Ying-Hai W (2006) Prisoner's dilemma game with nonlinear attractive effect on regular small-world networks. Chin Phys Lett 23(10):2874-2877

Jun W, Yue-Jin T, Hong-Zhong D, Da-Zhi Z (2008) In: Minai A, Bar-Yam Y, Braha D (ed) A new measure of heterogeneity of complex networks based on degree sequence. Springer, Berlin, $\mathrm{p} 66-73$

Karimi-Busheri F, Rasouli-Nia A (2015) In: Karimi-Busheri F (ed) Integration, networking, and global biobanking in the age of new biology. Springer International Publishing, Cham, pp 1-9

Kim B, Trusina A, Holme P, Minnhagen P, Chung J, Choi M (2002) Dynamic instabilities induced by asymmetric influence: prisoners' dilemma game in small-world networks. Phys Rev E 66(2, 1):021907

Knorr-Cetina K (1999) Epistemic cultures: how the sciences make knowledge. MIT Press, Cambridge

Leite D, Pinho I (2017) In: Theoretical approaches to research collaboration networks. Springer International Publishing, Cham, pp 25-39

Li X, Cao L (2003) Largest Laplacian eigenvalue predicts the emergence of costly punishment in the evolutionary ultimatum game on networks. Phys Rev E 80:066101-1-066101-6

Li P, Duan H (2014) Robustness of cooperation on scale-free networks in the evolutionary prisoner's dilemma game. EPL 105(4):48003

Liu RR, Rong Z, Jia CX, Wang BH (2010) Effects of diverse inertia on scale-free-networked prisoner's dilemma games. EPL 91(2):20002

Li A, Yong X (2015) Emergence of super cooperation of prisoner's dilemma games on scale-free networks. PLoS ONE 10(2):e0116429

Mali F, Kronegger L, Doreian P, Ferligoj A (2012) Dynamic scientific co-authorship networks. In: Scharnhorst A et al (eds) Models of science dynamics, understanding complex systems series. Springer, Berlin, pp 195-232

Masuda N, Aihara K (2003) Spatial prisoner's dilemma optimally played in small-world networks. Phys Lett A 313(1-2):55-61

Merton RK (1968) The Matthew effect in science. Science 159(3810):56-63

Motta-Murguia L, Saruwatari-Zavala G (2016) Mexican regulation of biobanks. J Law Med Ethics 44(1):58-67

Müller-Wille S, Charmantier I (2012) Natural history and information overload: the case of Linnaeus. Stud Hist Philos Biol Biomed Sci 43:4-15 
Newman MEJ (2001) Clustering and preferential attachment in growing networks. Phys Rev E 64:025102-1-025102-4 Newman MEJ (2004) Coauthorship networks and patterns of scientific collaboration. PNAS 101(suppl 1):5200-5205

Nowak MA, May RM (1992) Evolutionary games and spatial chaos. Nature 359:826-829

Oshtuki H, Hauert C, Lieberman E, Nowak MA (2006) A simple rule for the evolution of cooperation on social graphs. Nature 441:502-505

Price DJ (1965) Networks of scientific papers. Science 149(3683):510-515

Santos FC, Pacheco JM, Lenaerts T (2006) Cooperation prevails when individuals adjust their social ties. PLoS Computat Biol 2(10):1284-1291

Santos FC, Pacheco JM (2005) Scale-free networks provide a unifying framework for the emergence of cooperation. Phys Rev Lett 95:098104-1-098104-4

Shannon P, Markiel A, Ozier O, Baliga N, Wang J, Ramage D et al (2003) Cytoscape: a software environment for integrated models of biomolecular interaction networks. Genome Res 13(11):2498-2504

Shuai JW, Lin H, Wu CX (2009) Individual's strategy characterized by local topology conditions in prisoner's dilemma on scale-free networks. Phys Stat Mech Appl 388(13):2750-2756

Simon HA (1955) On a class of skew distribution functions. Biometrika 42(3-4):425-440

Strasser BJ (2006) Collecting and experimenting: the moral economies of biological research, 1960s-1980s. Preprint no 310. Max Planck Institute for the History of Science, Berlin

Strasser BJ (2012) Data-driven sciences: from wonder cabinets to electronic databases. Stud Hist Philos Biol Biomed Sci 43:85-87

Suri S, Watts DJ (2011) Cooperation and contagion in web-based. Networked public goods experiments. PLOS ONE 03:6

Szabó G, Fáth G (2007) Evolutionary games on graphs. Phys Rep 446:97-216

Szolnoki A, Perc M, Danku Z (2008) Towards effective payoffs in the prisoner's dilemma game on scale-free networks. Phys Stat Mech Appl 387(8-9):2075-2082

Thibert-Plante X, Parrott L (2007) Prisoner's dilemma and clusters on small-world networks. Complexity 12(6):22

Todorov R, Winterhager M (1991) An overview of Mike Moravcsik's publication activity in physics. Scientometrics 20(1):163-172

Turchin P (2015) Ultrasociety: how 10,000 years of war made humans the greatest cooperators on Earth

Vallance P, Freeman A, Stewart M (2016) Data sharing as part of the normal scientific process: a view from the pharmaceutical industry. PLOS Med 01:13

Vermeulen N, Parker JN, Penders B (2013) Understanding life together: a brief history of collaboration in biology. Endeavour 37:162-171

Wagner CS, Whetsell TA, Leydesdorff $L$ (2017) Growth of international collaboration in science: revisiting six specialties. Scientometrics 110(3):1633-1652

Wardil L, Hauert C (2015) Cooperation and coauthorship in scientific publishing. Phys Rev E 91:012825

Warner A, Moore H, Reinhard D, Ball L, Knoppers B (2016) Harmonizing global biospecimen consent practices to advance translational research: a call to action. Clin Pharmacol Ther 101(3)

Watts DJ (1999) Small worlds: the dynamics of networks between order and randomness. Princeton University Press, Princeton

Watts DJ, Strogatz SH (1998) Collective dynamics of 'small-world' networks. Nature 393:440-442

Wu ZX, Xu XJ, Chen Y, Wang YH (2005) Spatial prisoner's dilemma game with volunteering in Newman-Watts small-world networks. Phys Rev E 71:037103

Wu Z, Xu X, Wang Y (2006) Prisoner's dilemma game with heterogeneous influential effect on regular small-world networks. Chin Phys Lett 23(3):531-534

Yousefi-Nooraie R, Akbari-Kamrani M, Hanneman RA, Etemadi A (2008) Association between co-authorship network and scientific productivity and impact indicators in academic medical research centers: a case study in Iran. Health Res Policy Syst 9(6):1478-4505

Zhang S, Song Z, Wang X, Zhou W (2007) Emergence of small-world networks via local interaction using prisoner's dilemma game. In: Proceedings of IEEE congress on evolutionary computation, vols 1-10. IEEE, Singapore, Sep $25-28$, p $3706+$

\section{Submit your manuscript to a SpringerOpen ${ }^{\circ}$ journal and benefit from:}

- Convenient online submission

Rigorous peer review

- Immediate publication on acceptance

- Open access: articles freely available online

- High visibility within the field

- Retaining the copyright to your article

Submit your next manuscript at $\boldsymbol{\triangleright}$ springeropen.com 